\title{
Análisis longitudinal de las dimensiones corporales en adolescentes de la Escuela Nacional de Ballet de Cuba
}

\author{
Hamlet Betancourt León ${ }^{a}$ y María Elena díaz Sánchez \\ ${ }^{a}$ Centro Nacional de Escuelas de Arte. Departamento Investigaciones y Desarrollo. Ciudad Habana. Cuba. \\ blnstituto de Nutrición e Higiene de los Alimentos de Cuba. Departamento de Nutrición Comunitaria. Ciudad Habana. Cuba.
}

\section{RESUMEN}

Introducción: Para los maestros de ballet tiene gran importancia conocer las características y los cambios de la figura de los bailarines de ballet durante su crecimiento y desarrollo. El objetivo de esta investigación es determinar las variaciones de las dimensiones corporales de los bailarines de la Escuela Nacional de Ballet en 2 momentos del proceso de crecimiento, maduración y desarrollo.

Material y método: Se estudiaron longitudinalmente 54 bailarinas y 40 bailarines, con edades entre 15 y 20 años. Se aplicó un protocolo antropométrico de 26 mediciones para determinar las dimensiones corporales.

Resultados: Los bailarines manifestaron incrementos significativos de peso y estatura; la velocidad de crecimiento en la estatura disminuye con la edad, para todos los grupos de edad de uno y otro sexo. Se registró un incremento estadísticamente significativo en estatura en las bailarinas a expensas de la extremidad inferior para todos los grupos de edad. No se encontró ninguna tendencia específica de variación en las circunferencias musculares no corregidas para los bailarines y bailarinas de esta investigación. La mayoría de los pliegues cutáneos de todas las bailarinas no disminuyeron significativamente de un año a otro, en tanto para los bailarines ningún pliegue cutáneo manifestó incrementos significativos en el estudio longitudinal.

PALABRAS CLAVE: Cineantropometría. Ballet. Danza. Adolescencia. Dimensiones corporales.

\begin{abstract}
Introduction: Ballet teachers kneed to know the characteristics and changes that take place in the physiques of ballet dancers during growth and development. The objective of this study was to determine body dimension changes in dancers in the Cuban National Ballet School at 2 moments of growth, maturation and development.

Method: We performed a longitudinal study of 54 girls and 40 boys aged between 15 and 20 years. To determine body dimensions, an anthropometric protocol of 26 measurements was applied.

Results: In both girls and boys and all age groups, weight and height significantly increased, while growth velocity decreased with age. In girls in all age groups, statistically significant increases in height were found due to the lower extremity. No specific changes in variations in uncorrected muscle circumferences were found in either sex. Most skinfolds did not significantly decrease from one year to the next in girls while none of the skinfolds significantly increased in boys during the study.
\end{abstract}

KEY WORDS: Kinanthropometry. Ballet. Dance. Adolescence. Body dimensions. 


\section{INTRODUCCIÓN}

Los estudios del crecimiento y de la maduración humana tienen un interés básico en el conocimiento de las variaciones biológicas del cuerpo humano. La cineantropometría estudia las variaciones individuales del tamaño, las proporciones, la composición y la forma del cuerpo en relación con los diferentes aspectos del movimiento humano ${ }^{1}$.

El aprendizaje del ballet es un proceso complejo que debe sustentarse no sólo en la apreciación, sino también en la cuantificación de múltiples características morfológicas y fisiológicas. En la valoración funcional del bailarín se debe incluir el estudio del perfil cineantropométrico por ser uno de los factores que influyen en el éxito técnico artístico, tanto desde el punto de vista fisiológico, como biomecánico y/o estético.

Las modificaciones que ejerce el entrenamiento físico del ballet sobre el crecimiento, la maduración y el desarrollo no son fácilmente cuantificables. Resulta muy difícil establecer una línea divisoria entre los cambios biológicos derivados del propio entrenamiento y los que son consecuencia directa del proceso de crecimiento y desarrollo ${ }^{2}$. Las discusiones en torno al papel estimulador-acelerador o potencialmente negativo del entrenamiento físico intenso sobre el crecimiento y la maduración de los niños y adolescentes son un tema de mucha actualidad y que genera controversia en la comunidad científica ${ }^{3,4}$.

Se ha señalado que el peso corporal varía en su composición, fundamentalmente en las fracciones de masa grasa y masa muscular, con el entrenamiento físico en dependencia del tipo, de la intensidad y de la duración del programa de acondicionamiento ${ }^{5,6}$. Harrison et $\mathrm{al}^{7}$ refieren que la actividad física regular no genera un incremento en la masa ósea. La práctica de actividad física sistemática es un elemento estimulante en el crecimiento e integridad de los tejidos esquelético y muscular $^{2,8}$.

Existen indicios acerca de las influencias negativas potenciales de un entrenamiento intensivo en el crecimiento y la maduración de atletas jóvenes de alto nivel ${ }^{9}$. Un entrenamiento físico intenso durante la infancia y la adolescencia femenina puede resultar en amenorreas primarias y mineralizaciones óseas no óptimas ${ }^{10}$.

En las poblaciones especializadas algunos factores influyen más en los procesos de crecimiento, de maduración y de desarrollo debido a la intensidad y a la frecuencia con que se interactúa con ellos. Las demandas físicas y emocionales sobre los niños estudiantes de ballet en crecimiento son probablemente las más intensas entre todas las formas de danza ${ }^{11}$. La magnitud del efecto depende además de otros factores inherentes al proceso de aprendizaje, como la edad biológica, el sexo, la preinstrucción y las potencialidades genéticas del individuo ${ }^{12}$.

El objetivo de esta investigación es determinar las variaciones de las dimensiones corporales de los bailarines de la Escuela Nacional de Ballet de Cuba en 2 momentos del proceso de crecimiento, de maduración y de desarrollo humano normal.

\section{MATERIAL Y MÉTODO}

Se realizó un estudio longitudinal en adolescentes estudiantes de la Escuela Nacional de Ballet de Cuba (ENB) en el mes de abril de los años 2002 y 2003.

El estudio inicial (abril de 2002) fue realizado a 125 bailarines (75 mujeres y 50 varones), y el total de estudiantes medidos representaba más del $99 \%$ del universo de estudio en ese momento, con edades comprendidas entre los 15 y los 19 años. En el estudio final (abril de 2003) se midieron un total de 94 bailarines (54 mujeres y 40 varones). El descenso del $25 \%$ de la muestra se debió fundamentalmente a la culminación de estudios en la ENB de 13 estudiantes y el bajo rendimiento tecnicoartístico de otros 16 .

En la tabla I se muestran las cantidades de estudiantes que conformaron la población de estudio, medidos en ambas ocasiones según la edad cronológica y el sexo. Se definieron 3 grupos de edad, identificados por números romanos en la tabla, tomando como referencia la edad cronológica de los bailarines en la primera medición.

Las mediciones antropométricas se realizaron siguiendo los procedimientos estandarizados propuestos por Lohman et a ${ }^{13}$. La batería antropométrica empleada comprendió 26 medidas que se refieren a continuación: peso, estatura, talla sentado, longitud extremidad superior. Diámetros: biacromial, bicrestal, húmero, fémur. Circunferencias: brazo relajado, brazo fleTabla I Estudiantes que conformaron la población
de estudio

\begin{tabular}{|ccccc|}
\hline Grupo de edad & \multicolumn{2}{c}{ Edad (años) } & Sexo & Sexo \\
\hline & 2002 & 2003 & femenino & masculino \\
\hline I & 15 & 16 & 25 & 16 \\
\hline II & 16 & 17 & 18 & 13 \\
\hline III & $17,18,19$ & $18,19,20$ & 11 & 11 \\
\hline Total & & & 54 & 40 \\
\hline
\end{tabular}


xionado, antebrazo, torácica normal, cintura, cadera, muslo medio, pierna máxima. Pliegues cutáneos: subescapular, tríceps, bíceps, antebrazo, cintura, supraespinal, suprailíaco, periumbilical, muslo medio, pierna medial.

El análisis estadístico se realizó con el paquete estadístico SPSS 10.5 para Windows. El test de Kolgomorov-Smirnov se realizó a todas las variables continuas para determinar si seguían una distribución normal debido al pequeño tamaño de muestra por grupo de edad en cada sexo. Todas las variables cumplieron la distribución normal.

Se determinó la media y la desviación estándar de todas las variables estudiadas según el sexo de los bailarines para cada medición. Se calculó el diferencial para todas las mediciones antropométricas a partir de la resta del valor en la medición de 2002 a la medición de 2003. Las comparaciones univariadas entre las mediciones, tomadas en los momentos del estudio, para cada grupo de edad se realizaron utilizando la prueba t de Student para datos apareados $(\mathrm{p}<0,05)$.

\section{Resultados}

\section{Peso y estatura}

Los valores y las variaciones anuales de todas las mediciones tomadas en este estudio para cada grupo de edad se reflejan en las tablas II, III y IV para el sexo femenino y en las tablas V, VI y VII para el sexo masculino.

Para todos los grupos de edad del sexo femenino el peso y la estatura fueron mayores y estadísticamente significativos de un año a otro. En la muestra analizada las bailarinas más pesadas fueron las del grupo de edad III en el segundo corte $(50,1$ $\pm 4,3 \mathrm{~kg}$ ). Los diferenciales medios del peso corporal refieren el mayor aumento para el grupo de edad II, siendo muy pequeño el cuantificado para el grupo de edad III.

En los varones se observa para todos los grupos de edad que el peso y la estatura fueron mayores significativamente de una medición a otra. Los diferenciales de estas variables disminuyen según aumenta el grupo de edad.

\section{Dimensiones óseas}

Las bailarinas del grupo de edad I refieren incrementos significativos en la longitud de la extremidad superior y el diámetro biacromial. En los otros grupos de edad de bailarinas no se obtuvieron generalmente modificaciones significativas en la talla sentada, en la longitud de la extremidad superior y en los diámetros. Otros valores con incrementos negativos quedan en el marco del error técnico de la medición.

Los varones del grupo de edad I presentaron valores mayores y significativos para todas las dimensiones óseas excepto para el diámetro del húmero. Para el grupo de edad II se obtuvieron diferencias significativas solamente para la talla sentada y los diámetros biacromial y bicrestal. En el grupo de edad III todas las variables se mantuvieron estables, excepto el diámetro del fémur, de un año a otro.

\section{Circunferencias}

Para las bailarinas de los grupos de edad I y III no se encontraron incrementos significativos en la mayoría de las circunferencias de una medición a otra. En el grupo de edad II se manifiestan aumentos significativos en la gran mayoría de estas medidas.

En los varones del grupo de edad I muchas de las circunferencias aumentaron en tamaño significativamente de un año a otro, con excepción de las circunferencias del muslo medio y de la cintura. El grupo de edad II se comporta de manera diferente, pues solamente la circunferencia de la cintura y la de cadera incrementaron su perímetro significativamente. Para el grupo de edad III se señalan aumentos significativos para 2 circunferencias: brazo flexionado y cadera.

\section{Pliegues cutáneos}

Los pliegues cutáneos en las bailarinas del grupo de edad I apenas variaron de una medición a otra. En las bailarinas del grupo de edad II se observan aumentos significativos para los pliegues cutáneos de las extremidades del tríceps, del muslo medio y de la pierna medial, en tanto la mayoría de los pliegues cutáneos del torso no cambiaron significativamente de un año a otro. Ninguno de los pliegues cutáneos del grupo de edad III varió significativamente de una medición a otra.

En los varones ningún pliegue cutáneo aumentó significativamente de un año a otro para los 3 grupos de edad. Los bailarines del grupo de edad I disminuyeron significativamente casi todos los pliegues cutáneos, excepto el pliegue del antebrazo. En el grupo de edad II no variaron significativamente los pliegues del antebrazo y el periumbilical, en tanto para el grupo de edad III tuvieron igual comportamiento los pliegues subescapular, del tríceps y periumbilical. 
TRABAJOS ORIGINALES

Tabla II Dimensiones corporales y comparaciones para el grupo de edad I de bailarinas de la Escuela Nacional de Ballet

\begin{tabular}{|c|c|c|c|c|}
\hline \multirow[t]{2}{*}{ Dimensiones corporales } & \multicolumn{4}{|c|}{ Femenino $(n=25)$} \\
\hline & 2002 & 2003 & Diferencial & t apareado \\
\hline & Media \pm DE & Media \pm DE & & Significación \\
\hline Peso & $46,7 \pm 4,3$ & $49,0 \pm 4,4$ & 2,3 & $*$ \\
\hline Estatura & $159,8 \pm 4,7$ & $|6|, 2 \pm 4,5$ & $\mathrm{I}, 4$ & $*$ \\
\hline Talla sentado & $83,5 \pm 3,6$ & $84,0 \pm 2,9$ & 0,52 & NS \\
\hline Longitud extremidad superior & $68,4 \pm 2,7$ & $68,8 \pm 3,0$ & 0,44 & $*$ \\
\hline Diámetro biacromial & $34,0 \pm 1,6$ & $34,6 \pm 1,5$ & 0,55 & $*$ \\
\hline Diámetro bicrestal & $25,8 \pm 1,5$ & $26,0 \pm 1,4$ & 0,28 & NS \\
\hline Diámetro húmero & $5,9 \pm 1,0$ & $5,8 \pm 0,21$ & $-0,09$ & NS \\
\hline Diámetro fémur & $8,6 \pm 0,34$ & $8,7 \pm 0,34$ & 0,10 & $*$ \\
\hline Circunferencia brazo relajado & $20,9 \pm 1,0$ & $21,0 \pm 1,2$ & 0,09 & NS \\
\hline Circunferencia antebrazo & $20,5 \pm 0,91$ & $20,4 \pm 0,92$ & 0,06 & NS \\
\hline Circunferencia brazo flexionado & $22,2 \pm 1,2$ & $22,5 \pm 1,3$ & 0,26 & NS \\
\hline Circunferencia torácica & $76,4 \pm 2,9$ & $76,9 \pm 3,4$ & 0,44 & NS \\
\hline Circunferencia cintura & $61,5 \pm 2,9$ & $62,0 \pm 3,3$ & 0,48 & NS \\
\hline Circunferencia cadera & $83,1 \pm 3,6$ & $85,6 \pm 3,7$ & 2,4 & $*$ \\
\hline Circunferencia muslo medio & $46,3 \pm 2,0$ & $46,8 \pm 2,5$ & 0,47 & NS \\
\hline Circunferencia pierna máxima & $32,4 \pm 1,6$ & $33,1 \pm 1,5$ & 0,61 & $*$ \\
\hline Pliegue subescapular & $9,3 \pm 2,1$ & $9,3 \pm 3,5$ & $-0,07$ & NS \\
\hline Pliegue cintura & $\mathrm{II}, 0 \pm 3,8$ & $\mathrm{II}, \mathrm{I} \pm 4,7$ & 0,04 & NS \\
\hline Pliegue supraespinal & $5,4 \pm 1,5$ & $5,2 \pm 2,1$ & $-0,19$ & NS \\
\hline Pliegue suprailíaco & $7,0 \pm 2,7$ & $7,2 \pm 3,9$ & 0,22 & NS \\
\hline Pliegue periumbilical & $9,8 \pm 2,9$ & $10,9 \pm 4,6$ & 1,2 & NS \\
\hline Pliegue tríceps & $10,3 \pm 2,5$ & $10,8 \pm 3,3$ & 0,57 & NS \\
\hline Pliegue bíceps & $5,8 \pm 1,6$ & $5,2 \pm 1,8$ & $-0,66$ & * \\
\hline Pliegue antebrazo & $5, I \pm 0,76$ & $4,9 \pm 0,92$ & $-0,18$ & NS \\
\hline Pliegue muslo medio & $19,8 \pm 6,0$ & $22,3 \pm 8,0$ & 2,5 & $*$ \\
\hline Pliegue pierna medial & $12,4 \pm 3,9$ & $13,2 \pm 5,6$ & 0,83 & NS \\
\hline
\end{tabular}

NS: no significativo. ${ }^{*} \mathrm{p}<0,05$.

\section{Discusión}

\section{Peso y estatura}

A partir de la edad cronológica de 16 años la Tabla cubana de talla-edad del sexo femenino ${ }^{14}$ expresa coincidencia en los valores de estatura para todas las edades. En este estu- dio se observaron incrementos pequeños, pero significativamente estadísticos, para las bailarinas de los grupos de edad II y III que no están en concordancia con lo anteriormente referido.

En un estudio transversal en esta población realizado en noviembre de 2001 se encontró que la mayoría de los bailari- 
TRABAJOS ORIGINALES

Tabla III Dimensiones corporales y comparaciones para el grupo de edad II de bailarinas de la Escuela Nacional de Ballet

\begin{tabular}{|c|c|c|c|c|}
\hline \multirow[t]{2}{*}{ Dimensiones corporales } & \multicolumn{4}{|c|}{ Femenino $(n=18)$} \\
\hline & 2002 & 2003 & Diferencial & $\mathrm{t}$ apareado \\
\hline & Media \pm DE & Media $\pm \mathrm{DE}$ & & Significación \\
\hline Peso & $45,3 \pm 4,6$ & $48,0 \pm 4,8$ & 2,7 & $*$ \\
\hline Estatura & $158,6 \pm 4,5$ & $159,5 \pm 4,4$ & 0,90 & $*$ \\
\hline Talla sentado & $83,8 \pm 1,8$ & $84,0 \pm 1,5$ & 0,21 & NS \\
\hline Longitud extremidad superior & $67,5 \pm 2,3$ & $67,5 \pm 2,2$ & 0 & NS \\
\hline Diámetro biacromial & $34,2 \pm 1,5$ & $34,4 \pm 1,6$ & 0,22 & NS \\
\hline Diámetro bicrestal & $25,9 \pm 1,3$ & $25,9 \pm 1, I$ & 0 & NS \\
\hline Diámetro húmero & $5,6 \pm 0,27$ & $5,6 \pm 0,26$ & 0,09 & NS \\
\hline Diámetro fémur & $8,4 \pm 0,35$ & $8,5 \pm 0,36$ & 0,11 & $*$ \\
\hline Circunferencia brazo relajado & $20,8 \pm 1,4$ & $21,0 \pm 1,4$ & 0,23 & NS \\
\hline Circunferencia antebrazo & $20,| \pm 0,9|$ & $20,2 \pm 1,1$ & 0,08 & NS \\
\hline Circunferencia brazo flexionado & $21,7 \pm 1,2$ & $22,4 \pm 1,3$ & 0,68 & $*$ \\
\hline Circunferencia torácica & $75,4 \pm 3,3$ & $76,4 \pm 2,8$ & 0,94 & $*$ \\
\hline Circunferencia cintura & $59,8 \pm 3,4$ & $60,8 \pm 3,1$ & $\mathrm{I}, \mathrm{I}$ & $*$ \\
\hline Circunferencia cadera & $82,2 \pm 3,5$ & $84,6 \pm 4,0$ & 2,4 & $*$ \\
\hline Circunferencia muslo medio & $46,6 \pm 2,8$ & $47,2 \pm 2,9$ & 0,58 & NS \\
\hline Circunferencia pierna máxima & $32,2 \pm 1,6$ & $32,8 \pm 1,8$ & 0,61 & $*$ \\
\hline Pliegue subescapular & $8,6 \pm 2,4$ & $8,3 \pm 2,4$ & $-0,29$ & NS \\
\hline Pliegue cintura & $9,5 \pm 3,4$ & $9,8 \pm 4,2$ & 0,32 & NS \\
\hline Pliegue supraespinal & $4,8 \pm 0,96$ & $4,5 \pm 1,0$ & $-0,27$ & NS \\
\hline Pliegue suprailíaco & $5,9 \pm 1,8$ & $5,7 \pm 2,0$ & $-0,21$ & NS \\
\hline Pliegue periumbilical & $8, I \pm 2,7$ & $8,7 \pm 3,2$ & $-0,58$ & $*$ \\
\hline Pliegue tríceps & $10,0 \pm 2,2$ & $11,2 \pm 2,2$ & $\mathrm{I}, \mathrm{I}$ & $*$ \\
\hline Pliegue bíceps & $5,0 \pm 1,3$ & $4,7 \pm 1,1$ & $-0,26$ & NS \\
\hline Pliegue antebrazo & $4,8 \pm 0,88$ & $4,7 \pm 0,81$ & $-0,18$ & $*$ \\
\hline Pliegue muslo medio & $|8| \pm 5,2$, & $20,6 \pm 5,6$ & 2,5 & $*$ \\
\hline Pliegue pierna medial & $\mid I, I \pm 3,4$ & $12,8 \pm 3,8$ & 1,7 & $*$ \\
\hline
\end{tabular}

NS: no significativo. $* p<0,05$.

nes de uno y otro sexo presentaban un desarrollo sexual típico de la adolescencia media y tardía ${ }^{15}$. López Blanco et al ${ }^{16}$ señalan en una muestra de niñas venezolanas que las maduradoras tardías aún continúan creciendo a los 17 años.

Se informa en un estudio longitudinal de bailarinas de ballet una disminución en la velocidad de crecimiento en el 16\% de las bailarinas durante la etapa prepuberal con relación a un grupo control ${ }^{17}$. Evidencias indirectas de posibles efectos negativos del entrenamiento sobre la estatura son señaladas en varios casos donde se refiere un crecimiento compensatorio significativo en períodos de bajos volúmenes e intensidad de la actividad física ${ }^{18}$.

Los incrementos en estatura en las bailarinas pueden reflejar un déficit del crecimiento en esta dimensión en la etapa pre- 
TRABAJOS ORIGINALES

Tabla IV Dimensiones corporales y comparaciones para el grupo de edad III de bailarinas de la Escuela Nacional de Ballet

\begin{tabular}{|c|c|c|c|c|}
\hline \multirow[t]{2}{*}{ Dimensiones corporales } & \multicolumn{4}{|c|}{ Femenino $(n=I I)$} \\
\hline & 2002 & 2003 & Diferencial & $\mathrm{t}$ apareado \\
\hline & Media \pm DE & Media \pm DE & & Significación \\
\hline Peso & $48,8 \pm 4,0$ & $50, I \pm 4,3$ & $\mathrm{I}, 4$ & $*$ \\
\hline Estatura & $160,0 \pm 5,3$ & $160,7 \pm 5,3$ & 0,68 & $*$ \\
\hline Talla sentado & $84,9 \pm 2,9$ & $84,5 \pm 3,2$ & $-0,35$ & NS \\
\hline Longitud extremidad superior & $68,0 \pm 2,6$ & $67,9 \pm 2,9$ & $-0,08$ & NS \\
\hline Diámetro biacromial & $34,5 \pm 1, I$ & $34,6 \pm 1,0$ & 0,09 & NS \\
\hline Diámetro bicrestal & $26,7 \pm 1,3$ & $26,3 \pm 1,0$ & $-0,39$ & NS \\
\hline Diámetro húmero & $5,6 \pm 0,22$ & $5,6 \pm 0,32$ & $-0,01$ & NS \\
\hline Diámetro fémur & $8,5 \pm 0,49$ & $8,5 \pm 0,41$ & $-0,01$ & NS \\
\hline Circunferencia brazo relajado & $21,6 \pm 1,3$ & $2 \mathrm{I}, 4 \pm \mathrm{I}, 4$ & $-0,27$ & NS \\
\hline Circunferencia antebrazo & $20,8 \pm 0,73$ & $20,6 \pm 1,0$ & $-0,24$ & NS \\
\hline Circunferencia brazo flexionado & $22,7 \pm 1,2$ & $22,9 \pm 1,5$ & 0,20 & NS \\
\hline Circunferencia torácica & $77,0 \pm 1,2$ & $77,5 \pm 2,7$ & 0,47 & NS \\
\hline Circunferencia cintura & $62,2 \pm 2,2$ & $62,5 \pm 2,5$ & 0,34 & NS \\
\hline Circunferencia cadera & $84,0 \pm 4, I$ & $85,7 \pm 3,9$ & $\mathrm{I}, 7$ & $*$ \\
\hline Circunferencia muslo medio & $47,7 \pm 2,7$ & $47,6 \pm 2,5$ & $-0,14$ & NS \\
\hline Circunferencia pierna máxima & $33,2 \pm 1,6$ & $33,3 \pm 1,3$ & 0,07 & NS \\
\hline Pliegue subescapular & $11,2 \pm 3,4$ & $10,5 \pm 3,1$ & $-0,71$ & NS \\
\hline Pliegue cintura & $14,8 \pm 6,5$ & $13,6 \pm 6,4$ & $-1,2$ & NS \\
\hline Pliegue supraespinal & $6,5 \pm 2,0$ & $5,9 \pm 2,6$ & $-0,54$ & NS \\
\hline Pliegue suprailíaco & $8,5 \pm 3,0$ & $7,8 \pm 3,5$ & $-0,66$ & NS \\
\hline Pliegue periumbilical & $10,7 \pm 5,1$ & $\mid I, I \pm 5,6$ & $-0,44$ & NS \\
\hline Pliegue tríceps & $1 \mathrm{I}, 4 \pm 3,4$ & $11,0 \pm 2,9$ & $-0,40$ & NS \\
\hline Pliegue bíceps & $5,9 \pm 2,2$ & $5,4 \pm 1,9$ & $-0,51$ & NS \\
\hline Pliegue antebrazo & $5,9 \pm 1,3$ & $5,40 \pm 1,0$ & $-0,47$ & NS \\
\hline Pliegue muslo medio & $20,7 \pm 8,3$ & $21,2 \pm 7,9$ & 0,54 & NS \\
\hline Pliegue pierna medial & $14,2 \pm 7,6$ & $|3| \pm 6,2$, & $-1,0$ & NS \\
\hline
\end{tabular}

NS: no significativo. ${ }^{p} p<0,05$.

puberal que está siendo compensado en la adolescencia tardía, o expresar el ritmo natural de crecimiento de la estatura para esta población especializada. Las integrantes de una población de bailarinas de alto nivel deben ser maduradoras tardías, pues esto constituiría una ventaja en términos cognitivos debido al papel negativo que los tempranos incrementos de grasa corpo- ral tienen sobre la linealidad de la figura y la amplitud y calidad de los movimientos.

Los resultados no expresan una tendencia definida de disminución o aumento de peso corporal para las bailarinas independiente de su edad cronológica de un año a otro. En los varones no se manifiesta la tendencia de mayor estatura 
TRABAJOS ORIGINALES

Tabla V Dimensiones corporales y comparaciones para el grupo de edad I de bailarines de la Escuela Nacional de Ballet

\begin{tabular}{|c|c|c|c|c|}
\hline \multirow[t]{2}{*}{ Dimensiones corporales } & \multicolumn{4}{|c|}{ Masculino $(n=16)$} \\
\hline & 2002 & 2003 & Diferencial & $\mathrm{t}$ apareado \\
\hline & Media \pm DE & Media $\pm \mathrm{DE}$ & & Significación \\
\hline Peso & $57,3 \pm 6,7$ & $60,9 \pm 5,3$ & 3,6 & $*$ \\
\hline Estatura & $\mid 70,1 \pm 5,2$ & $173,2 \pm 4,7$ & 3,2 & $*$ \\
\hline Talla sentado & $88,4 \pm 3,5$ & $90,0 \pm 3,0$ & 1,5 & $*$ \\
\hline Longitud extremidad superior & $72,8 \pm 2,8$ & $74,4 \pm 2,5$ & 1,6 & $*$ \\
\hline Diámetro biacromial & $37,4 \pm 2,1$ & $38,7 \pm 1,4$ & 1,3 & $*$ \\
\hline Diámetro bicrestal & $26,2 \pm 1,3$ & $27, I \pm 1,2$ & 0,96 & $*$ \\
\hline Diámetro húmero & $6,8 \pm 0,36$ & $6,8 \pm 0,28$ & 0,04 & NS \\
\hline Diámetro fémur & $9,7 \pm 0,42$ & $9,9 \pm 0,43$ & 0,19 & $*$ \\
\hline Circunferencia brazo relajado & $24,7 \pm 2,2$ & $25,5 \pm 1,9$ & 0,83 & $*$ \\
\hline Circunferencia antebrazo & $24,1 \pm 1,2$ & $24,5 \pm 0,95$ & 0,46 & $*$ \\
\hline Circunferencia brazo flexionado & $27,0 \pm 2,2$ & $28,1 \pm 1,8$ & $\mathrm{I}, \mathrm{I}$ & * \\
\hline Circunferencia torácica & $84,3 \pm 5,0$ & $86,6 \pm 4,4$ & 2,4 & $*$ \\
\hline Circunferencia cintura & $68,0 \pm 3,3$ & $68,5 \pm 2,0$ & 0,49 & NS \\
\hline Circunferencia cadera & $83,7 \pm 3,6$ & $86,0 \pm 3,0$ & 2,2 & $*$ \\
\hline Circunferencia muslo medio & $48,6 \pm 2,7$ & $49,1 \pm 1,9$ & 0,53 & NS \\
\hline Circunferencia pierna máxima & $34,8 \pm 1,5$ & $35,5 \pm 1,0$ & 0,67 & $*$ \\
\hline Pliegue subescapular & $7,9 \pm 1,6$ & $7,5 \pm 1,5$ & $-0,39$ & $*$ \\
\hline Pliegue cintura & $8,4 \pm 1,8$ & $7,0 \pm 1,1$ & $-I, 4$ & $*$ \\
\hline Pliegue supraespinal & $4,4 \pm 0,81$ & $3,9 \pm 0,49$ & $-0,54$ & $*$ \\
\hline Pliegue suprailíaco & $5,7 \pm I, I$ & $4,8 \pm 0,71$ & $-0,91$ & $*$ \\
\hline Pliegue periumbilical & $7,2 \pm 1,6$ & $6,8 \pm 1,4$ & $-0,47$ & $*$ \\
\hline Pliegue tríceps & $7,0 \pm 1,7$ & $6,3 \pm 1,9$ & $-0,69$ & $*$ \\
\hline Pliegue bíceps & $4,1 \pm 0,63$ & $3,6 \pm 0,76$ & $-0,48$ & $*$ \\
\hline Pliegue antebrazo & $5, I \pm 0,75$ & $4,9 \pm 0,76$ & $-0,12$ & NS \\
\hline Pliegue muslo medio & $9,9 \pm 2,1$ & $8,6 \pm 2,4$ & $-1,3$ & $*$ \\
\hline Pliegue pierna medial & $7,4 \pm 1,3$ & $6,4 \pm 1,4$ & $-0,99$ & $*$ \\
\hline
\end{tabular}

NS: no significativo. ${ }^{*} \mathrm{p}<0,05$.

según la edad cronológica y menor peso corporal según la estatura.

En algunas actividades, especialmente en deportes estéticos y danza, un incremento en el peso corporal puede tener una influencia negativa en el rendimiento ${ }^{19}$. Resulta necesario estudiar la variación individual y por grupo de edad del peso, teniendo en cuenta su composición, asociada a evaluaciones técnico-artísticas realizadas por los maestros de ballet que comprendan sus criterios de gordura-linealidad de la figura de la bailarina.

Las velocidades de crecimiento de estatura en los varones son siempre mayores que las de las hembras en todos los grupos de edad. El carácter selectivo de ingreso a la población de bailarines adolescentes de ballet no está relacionado directamente 
TRABAJOS ORIGINALES

Tabla VI Dimensiones corporales y comparaciones para el grupo de edad II de bailarines de la Escuela Nacional de Ballet

\begin{tabular}{|c|c|c|c|c|}
\hline \multirow[t]{2}{*}{ Dimensiones corporales } & \multicolumn{4}{|c|}{ Masculino $(n=13)$} \\
\hline & 2002 & 2003 & Diferencial & $\mathbf{t}$ apareado \\
\hline & Media $\pm \mathrm{DE}$ & Media $\pm \mathrm{DE}$ & & Significación \\
\hline Peso & $59,6 \pm 5,3$ & $62,2 \pm 6,4$ & 2,6 & $*$ \\
\hline Estatura & $170,3 \pm 4,9$ & $172,3 \pm 4,7$ & 2,0 & $*$ \\
\hline Talla sentado & $89,7 \pm 2,7$ & $90,5 \pm 2,3$ & 0,86 & $*$ \\
\hline Longitud extremidad superior & $74,6 \pm 3,0$ & $75,0 \pm 3,2$ & 0,39 & NS \\
\hline Diámetro biacromial & $38, I \pm I, 8$ & $38,9 \pm 1,6$ & 0,81 & $*$ \\
\hline Diámetro bicrestal & $26,6 \pm 0,87$ & $27,0 \pm 0,75$ & 0,40 & $*$ \\
\hline Diámetro húmero & $6,9 \pm 0,32$ & $6,9 \pm 0,32$ & 0,03 & NS \\
\hline Diámetro fémur & $9,9 \pm 0,29$ & $9,8 \pm 0,28$ & 0,06 & NS \\
\hline Circunferencia brazo relajado & $25,4 \pm 1,5$ & $25,8 \pm 1,7$ & 0,41 & NS \\
\hline Circunferencia antebrazo & $24,6 \pm 1,2$ & $24,9 \pm 1,3$ & 0,29 & NS \\
\hline Circunferencia brazo flexionado & $27,9 \pm 1,8$ & $28,6 \pm 1,8$ & 0,69 & NS \\
\hline Circunferencia torácica & $87,2 \pm 2,9$ & $88,0 \pm 3,6$ & 0,80 & NS \\
\hline Circunferencia cintura & $67,4 \pm 3,4$ & $68,8 \pm 3,2$ & 1,5 & $*$ \\
\hline Circunferencia cadera & $85,2 \pm 2,8$ & $87,1 \pm 4,2$ & 1,9 & $*$ \\
\hline Circunferencia muslo medio & $50,5 \pm 3,2$ & $5 \mathrm{I}, 4 \pm 3,6$ & 0,95 & NS \\
\hline Circunferencia pierna máxima & $36,2 \pm 1,8$ & $36,3 \pm 1,8$ & 0,16 & NS \\
\hline Pliegue subescapular & $7,3 \pm 0,54$ & $6,9 \pm 0,46$ & $-0,38$ & $*$ \\
\hline Pliegue cintura & $7,4 \pm 1,5$ & $6,6 \pm 1,1$ & $-0,82$ & $*$ \\
\hline Pliegue supraespinal & $4,5 \pm 0,44$ & $4,0 \pm 0,41$ & $-0,49$ & $*$ \\
\hline Pliegue suprailíaco & $5,5 \pm 0,81$ & $4,8 \pm 0,66$ & $-0,72$ & $*$ \\
\hline Pliegue periumbilical & $6,7 \pm 1,0$ & $6,6 \pm 1,3$ & $-0,12$ & NS \\
\hline Pliegue tríceps & $6,7 \pm 0,98$ & $5,8 \pm 0,85$ & $-0,95$ & $*$ \\
\hline Pliegue bíceps & $3,8 \pm 0,36$ & $3,3 \pm 0,41$ & $-0,42$ & $*$ \\
\hline Pliegue antebrazo & $4,9 \pm 0,64$ & $4,6 \pm 0,78$ & $-0,35$ & NS \\
\hline Pliegue muslo medio & $9,6 \pm 2,2$ & $7,8 \pm 1,8$ & $-1,8$ & $*$ \\
\hline Pliegue pierna medial & $7,3 \pm 1,5$ & $5,9 \pm 1,2$ & $-1,4$ & $*$ \\
\hline
\end{tabular}

NS: no significativo. $* p<0,05$.

con las potencialidades de crecimiento o la estatura alcanzada en un momento determinado. Actualmente en la ENB una estatura baja no constituye un obstáculo para el ingreso de un bailarín; los maestros de ballet siempre tienen la esperanza del estirón en los niños buenos técnicamente, pero pequeños en estatura.

Los bailarines manifestaron incrementos significativos de peso y talla para todos los grupos de edad de uno y otro sexo, y la velocidad de crecimiento de la estatura disminuye con la edad.

\section{Dimensiones óseas}

La invariabilidad significativa de la talla sentada en las bailarinas explica que el crecimiento significativo en estatura en 
TRABAJOS ORIGINALES

Tabla VII Dimensiones corporales y comparaciones para el grupo de edad III de bailarines de la Escuela Nacional de Ballet

\begin{tabular}{|c|c|c|c|c|}
\hline \multirow[t]{2}{*}{ Dimensiones corporales } & \multicolumn{4}{|c|}{ Masculino $(n=I I)$} \\
\hline & 2002 & 2003 & Diferencial & $\mathrm{t}$ apareado \\
\hline & Media \pm DE & Media $\pm \mathrm{DE}$ & & Significación \\
\hline Peso & $60,5 \pm 2,9$ & $62,2 \pm 2,0$ & 1,6 & $*$ \\
\hline Estatura & $173,6 \pm 2,0$ & $174,5 \pm 2,1$ & 0,92 & $*$ \\
\hline Talla sentado & $90,8 \pm 2,2$ & $91,2 \pm 2,0$ & 0,38 & NS \\
\hline Longitud extremidad superior & $75,4 \pm 1,6$ & $75,4 \pm 1,7$ & $-0,04$ & NS \\
\hline Diámetro biacromial & $39,4 \pm 1,2$ & $39,7 \pm 1,3$ & 0,30 & NS \\
\hline Diámetro bicrestal & $26,7 \pm 1, I$ & $26,9 \pm 1,1$ & 0,16 & NS \\
\hline Diámetro húmero & $6,7 \pm 0,40$ & $6,8 \pm 0,28$ & 0,04 & NS \\
\hline Diámetro fémur & $9,6 \pm 0,33$ & $9,7 \pm 0,38$ & 0,13 & $*$ \\
\hline Circunferencia brazo relajado & $25,9 \pm 1,8$ & $26,2 \pm 1,4$ & 0,25 & NS \\
\hline Circunferencia antebrazo & $24,8 \pm 1,2$ & $24,8 \pm 1,1$ & $-0,05$ & NS \\
\hline Circunferencia brazo flexionado & $28,0 \pm 1,5$ & $28,8 \pm 1,4$ & 0,81 & $*$ \\
\hline Circunferencia torácica & $87,6 \pm 3,5$ & $88,3 \pm 3,5$ & 0,72 & NS \\
\hline Circunferencia cintura & $69,2 \pm 3,4$ & $70,4 \pm 1,9$ & $\mathrm{I}, 2$ & NS \\
\hline Circunferencia cadera & $84,3 \pm 2,2$ & $85,6 \pm 2,0$ & $\mathrm{I}, 3$ & $*$ \\
\hline Circunferencia muslo medio & $49,9 \pm 1,8$ & $49,8 \pm 1,6$ & $-0,07$ & NS \\
\hline Circunferencia pierna máxima & $35,7 \pm 0,94$ & $35,9 \pm 0,67$ & 0,19 & NS \\
\hline Pliegue subescapular & $8,4 \pm 1,4$ & $8,0 \pm 1,3$ & $-0,46$ & NS \\
\hline Pliegue cintura & $8,0 \pm 1,4$ & $7,3 \pm 1,3$ & $-0,69$ & $*$ \\
\hline Pliegue supraespinal & $4,8 \pm 1, I$ & $4,0 \pm 0,94$ & $-0,73$ & $*$ \\
\hline Pliegue suprailíaco & $6,0 \pm 1,5$ & $5,0 \pm 1,2$ & $-1,0$ & $*$ \\
\hline Pliegue periumbilical & $7,7 \pm 1,9$ & $7,3 \pm 1,9$ & $-0,36$ & NS \\
\hline Pliegue tríceps & $6,7 \pm 1,6$ & $6,3 \pm 1,6$ & $-0,34$ & NS \\
\hline Pliegue bíceps & $3,8 \pm 0,60$ & $3,5 \pm 0,70$ & $-0,3$ I & $*$ \\
\hline Pliegue antebrazo & $5,1 \pm 0,63$ & $4,7 \pm 0,71$ & $-0,46$ & $*$ \\
\hline Pliegue muslo medio & $9,4 \pm 2,5$ & $8,5 \pm 2,8$ & $-0,89$ & $*$ \\
\hline Pliegue pierna medial & $6,5 \pm 1,4$ & $5,9 \pm 1,4$ & $-0,56$ & $*$ \\
\hline
\end{tabular}

NS: no significativo. ${ }^{*} p<0,05$.

los 3 grupos de edad durante la adolescencia tardía está ocurriendo fundamentalmente a expensas del aumento de la extremidad inferior. Con el incremento significativo de la estatura las bailarinas tendieron a disminuir proporcionalmente el tamaño de su extremidad superior; esto afecta la estética de su figura y no favorece la ejecución de algunas tareas técnicas característica del ballet.
La especie humana tiene un patrón básico de crecimiento y maduración, variable, modulado e independiente de disímiles factores intrínsecos y extrínsecos ${ }^{3}$. Como han señalado Bass et $\mathrm{al}^{20}$, el crecimiento en estatura preadolescente ocurre fundamentalmente a expensas de la extremidad inferior, mientras el crecimiento en la pubertad se caracteriza por una aceleración de la longitud del tronco. 
Si pensamos que las bailarinas cubanas son seleccionadas a los 9 años si presentan el torso estrecho y las piernas largas, entre más de 20 características somatológicas evaluadas cualitativamente, entonces empíricamente se están escogiendo generalmente maduradoras tardías o maduradoras promedio con fenotipo de piernas largas. No obstante, en bailarinas maduradoras tardías, si no se varía el patrón de crecimiento humano normal, se debe cuantificar el mismo gradiente de crecimiento que en las maduradoras tempranas o promedio, pues el crecimiento, la maduración y el desarrollo son procesos sumamente organizados, aunque estén caracterizados por variaciones individuales 5 .

Otras investigaciones han obtenido evidencias similares a la de este estudio longitudinal en poblaciones de bailarinas de ballet y gimnastas. Warren ${ }^{21}$ describe deficiencias relativas en el crecimiento del tronco con relación a las extremidades inferiores en bailarinas de ballet. Bass et $\mathrm{a}^{22}$ señalan que el entrenamiento físico intensivo durante la pubertad disminuye significativamente la velocidad de crecimiento del tronco en gimnastas de alto nivel comparadas con un grupo control. Otra investigación sugiere que el entrenamiento intensivo, combinado con una ingesta nutricional deficiente, puede alterar el normal desarrollo puberal de los individuos expuestos ${ }^{23}$.

El incremento significativo en estatura en las bailarinas ocurre a expensas de la extremidad inferior para todos los grupos de edad. En general se observa una reducción en la velocidad de incremento para estas dimensiones óseas que se expresa en la disminución de los diferenciales a medida que los adolescentes cambian de edad. De un año a otro los incrementos de las longitudes y diámetros ocurrieron en menos dimensiones óseas, a medida que aumentó la edad en uno y otro sexo.

\section{Circunferencias}

En el análisis de las circunferencias musculares no corregidas se observaron diferentes tendencias para cada grupo de edad en las bailarinas. No obstante, el comportamiento de los diferenciales en las circunferencias sigue la tendencia de disminuir, ya que los incrementos son muy pequeños, a medida que las bailarinas aumentan su edad cronológica. En los bailarines no se observan tendencias definidas de incremento del valor de las circunferencias en correspondencia con los grupos de edad, así como tampoco de los diferenciales correspondientes.

El incremento en peso corporal de un año a otro, referido anteriormente, se expresa en el aumento de algunas de las dimensiones óseas y de los tejidos blandos medidos. Este aumento significativo en algunas circunferencias, principalmente de la cadera, del muslo medio y de la pierna máxima, no se correlaciona directamente con el aumento de la fuerza, ni relativa ni absoluta, con la velocidad de movimiento o con la flexibilidad de esos segmentos. Resulta necesario determinar el aporte de la mineralización ósea, imposible por la antropometría, y la deposición grasa para predecir, a partir de la medición fisiológica, las variaciones de estas capacidades físicas $^{24}$.

La adquisición de las habilidades no está relacionada directamente con el aumento de volumen muscular de todos los segmentos. La forma del muslo en el ballet es decisiva en la apreciación de la figura y expresa la calidad del trabajo académico del bailarín. La correcta realización de la técnica crea un muslo elongado, fuerte en su sección medial interna, con un desarrollo limitado del volumen del cuádriceps para permitir estirar la pierna en flexión plantar máxima.

En el ballet se necesita que el tamaño, el volumen y la forma de las caderas de las mujeres siga un patrón andrógeno, no sólo por su valor estético, sino también por las ventajas que ofrece en la ejecución de la técnica artística. Las evidencias de similitud entre los sexos para la circunferencia de la cadera están dadas por los altos valores de las hembras relativos a su estatura, lo cual coincide con los datos referidos en estudios de crecimiento y desarrollo en poblaciones normales $^{14,25}$. Teniendo en cuenta las características de la figura de la bailarina y las diferencias en estatura a favor de los varones en este estudio, las bailarinas debieron reflejar valores promedios menores de circunferencia de la cadera que los obtenidos.

No se encontró ninguna tendencia específica de variación en las circunferencias musculares no corregidas para los bailarines y bailarinas de esta investigación.

\section{Pliegues cutáneos}

Los incrementos de peso corporal no ocurrieron fundamentalmente a expensas del tejido graso para las bailarinas de los grupos de edad I y III, lo que expresa una disminución relativa de las cantidades de masa grasa para un peso determinado. Las bailarinas del grupo de edad II manifiestan la búsqueda del patrón ginecoide de las adolescentes en estas edades para una población normal, pues algunos pliegues de las extremidades muestran incrementos, y valores absolutos, mayores significativamente que los pliegues del torso de un año a otro $^{26}$. 
En los bailarines la disminución del grosor de todos los pliegues cutáneos de un año a otro señala un incremento de la masa corporal a expensas de otros tejidos, lo cual expresa una disminución porcentual de la cantidad de tejido graso subcutáneo. Los bailarines de esta población no manifiestan el patrón biológico de distribución de los depósitos de la grasa corporal en adolescentes de una población normal. Baxter-Jones et al ${ }^{3}$ plantean que en los varones generalmente los pliegues cutáneos de las extremidades disminuyen durante la adolescencia normal, mientras los pliegues del tronco no siguen esta tendencia.

La mayoría de los pliegues cutáneos de todas las bailarinas no disminuyeron significativamente de un año a otro, en tanto que para los bailarines ningún pliegue cutáneo manifestó incrementos significativos en el estudio longitudinal.

\section{Bibliografía}

1. Ross W, Carr R, Carter L. Anthropometry ilustrated. Canadá: Turnpike Electronic Publications Inc.; 1999.

2. Pérez BM. Efectos del entrenamiento sobre el crecimiento y desarrollo en niños y adolescentes. Tribuna del Investigador. 1997; 4:102-11.

3. Baxter-Jones AD, Thompson AM, Malina RM. Growth and maturation in elite young female athletes. Sports Med and Arthroscopy Review. 2002;10:42-9.

4. Daly RM, Bass S, Caine D, Howe W. Does training affect growth? Answers to common questions. The Physician and Sportsmedicine. 2002;30:1-12.

5. Malina RM, Bouchard C. Growth, maturation and physical activity. Champaign, Illinois: Human Kinetics Books; 1991.

6. Damsgaard R. Children in competitive sports. Growth, pubertal development, body proportions, body composition, endocrinology, bone mineralization and nutrition. Disponible en: http:// www. doping.dk/db/filarkiv/55/pdfstar.pdf

7. Harrison G, Tanner J, Pilbeam D, Baker P. Human biology. New York: Cambridge University Press; 1993.

8. Rich P. Growth: intense training in prepubertal children. Disponible en: http://www.sportsci.org/encyc/drafts/child_growth_ intense_train.doc

9. Malina RM. Sports and children. Asia-Pacific J. 1998;133-8.

10. Bass S, Pearce G, Young N, Seeman E. Bone mass during growth: the effects of exercise. Exercise and mineral accrual. Acta Univ Caro. 1994;40:3-6.

11. Wan M, Wing W, Chan KM. Dance medicine. Women athletes. 2002;161-7.

12. Berk LE. Infants, children and adolescents. Boston: Allyn \& Bacon; 1999.

13. Lohman TG, Roche AF, Martorell R. Anthropometric standardization reference manual. Champaign, Illinois: Human Kinetic Publishers; 1988.

14. Jordan JR. Desarrollo humano en Cuba. La Habana: Científico Técnica; 1979.

15. Díaz ME, Reboso J, Martínez A, Toledo E, Wong I, Moreno V, et al. Desarrollo físico y estado nutricional en estudiantes de ba- llet. Ponencia en el VII Simposio de Antropología Física Luis Montané. Universidad de la Habana. Febrero de 2003.

16. López Blanco M, Hernández de Valera Y, Landaeta M, Henríquez G. Crecimiento y nutrición en la región latinoamericana. Anales Venezolanos de Nutrición. 1993;6:47-90.

17. Pigeon P, Oliver I, Charlet JP, et al. Intensive dance practice: repercussions on growth and puberty. Am J Sports Me. 1997;25: 243-7.

18. Tveit-Milligan P, Spindler AA, Nichols JF. Genes and gymnastics: a case study of triplets. Sports Med Train Rehab. 1993;4:47-52.

19. Wilmore JH. Body weight standards and athletic performance. En: Rownell KD, Rodin J, Wilmore JH, editores. Eating, body weight and performance in athletes: disorders of modern society. Philadelphia: Lea and Febiger; 1992. p. 315-29.

20. Bass S, Delmas PD, Pearce G, et al. The differing tempo of growth in bone size, mass, and density in girls is region-specific. J Clin Invest. 1999;104:795-804.

21. Warren MP. The effects of exercise on pubertal progression and reproductive function in girls. J Clin Endocrinol Metab. 1980; 51: 1150-7.

22. Bass S, Bradney M, Pearce G, et al. Short stature and delayed puberty in gymnasts: influence of selection bias on leg length and the duration of training on trunk length. J Pediatr. 2000;136: 149-55.

23. Weimann E, Witzel C, Schwidergall S, et al. Peripubertal perturbations in elite gymnasts caused by sport specific training regimes and inadequate nutritional intake. Int J Sports Med. 2000; 21:210-5.

24. Sale DG. Testing strength and power. En: Wenger J, Green HJ, editors. Physiological testing of the high-performance athlete. Champaign, Illinois: Human Kinetics; 1991. p. 21-106.

25. Méndez Castellanos H, editor. Crecimiento físico y maduración. En: Estudio Nacional de Crecimiento y Desarrollo Humanos de la República de Venezuela. Caracas: Fundacredesa; 1995. p. 407-609.

26. Mueller WH. The biology of human fat patterning. En: Norgan NG, editor. Human body composition and fat distribution. London: Euro-Nut; 1985. p. 160-74. 